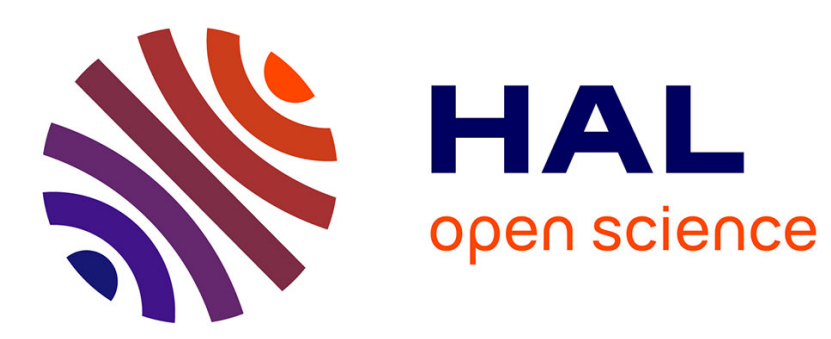

\title{
Aide in decision-making: contribution to uncertainties in three-dimensional measurement
}

Jérôme Bachmann, Jean-Marc Linares, Jean Michel Sprauel, Pierre Bourdet

\section{To cite this version:}

Jérôme Bachmann, Jean-Marc Linares, Jean Michel Sprauel, Pierre Bourdet. Aide in decision-making: contribution to uncertainties in three-dimensional measurement. Precision Engineering, 2004, 28, pp.78 - 88. 10.1016/S0141-6359(03)00079-5 . hal-01410084

\section{HAL Id: hal-01410084 \\ https://hal-amu.archives-ouvertes.fr/hal-01410084}

Submitted on 7 Dec 2016

HAL is a multi-disciplinary open access archive for the deposit and dissemination of scientific research documents, whether they are published or not. The documents may come from teaching and research institutions in France or abroad, or from public or private research centers.
L'archive ouverte pluridisciplinaire HAL, est destinée au dépôt et à la diffusion de documents scientifiques de niveau recherche, publiés ou non, émanant des établissements d'enseignement et de recherche français ou étrangers, des laboratoires publics ou privés. 


\title{
Aide in decision-making: contribution to uncertainties in three-dimensional measurement
}

\author{
Jérome Bachmann $^{\mathrm{a}, *}$, Jean marc Linares ${ }^{\mathrm{a}}$, Jean Michel Sprauel ${ }^{\mathrm{a}}$, Pierre Bourdet ${ }^{\mathrm{b}}$ \\ ${ }^{a}$ EAMS, Inst. Univ. de Technologie (IUT), Université de la Méditerranée, Avenue Gaston Berger, F13625 Aix en Provence Cedex 1, France ${ }^{\mathrm{b}}$ \\ LURPA, ENS Cachan, Avenue du Président Wilson, F94230 Cachan, France
}

\begin{abstract}
The authorities of the standards organization International Organization of Standardization (ISO) advocate mastering any uncertainties in all parts of the industrialization process. In the three-dimensional (3D) measurement process, uncertainty is usually obtained at the end of a battery of tests. It is defined as a whole because it includes several types of errors, known systematic components, unknown systematic components and random components. Automated calculations of uncertainty can be made based on statistics. This method is based on statistical concepts, which are in accordance with "The Guide to the expression of the uncertainty in measurement" (GUM). It also enables us to generate uncertainties on the verification of ISO specifications (or specs in the ISO directives). In the course of this article, a usage will be presented that takes the knowledge of uncertainties into account: this usage will help the operator to take a decision on the conformance of a mechanical part in reference to its conformance to geometric tolerance.
\end{abstract}

Keywords: Uncertainties; Propagation; ISO tolerances

\section{Introduction}

One of the concerns of the ISO/TC 213 [1] work group is to take uncertainties into account in the global industrialization process. It is a question of mastering the propagation of the uncertainty due to best-fit calculations and geometric constructions that the metrologist makes while checking specifications. This objective must be met while respecting the duality principle according to which it is difficult to specify a geometric function without integrating verification procedures [2].

In the past few years, the group has attempted to reduce any deficiencies and contradictions which affected dimensional and geometric tolerance. Proposals were then adapted to the functions of the part. The tools proposed could not be applied to mechanical specifications. Up to now, the explanation of standards has been illustrated by a certain number of unique cases. Today, ISO/TC 213 is moving towards a univocal and generic mathematical description of the problems of geometric specifications [3].

On a larger scale, The Geometric Product Specification (GPS; ISO/TC 14638) matrix has been defined, and it aims at creating a coherent system for the elaboration of standards for the industrialization of a product. It can coherently define a set of standards in relation to a particular problem and to the geometric characteristics of the element. Among the standards suggested, GPS language provides a transverse response to the problems of checking and specifying. However, some deficiencies are observed in tolerance formalization. In this context, ISO/TC 213 proposes a concept based on a mathematical formalism, which can express any dimensional and geometric demand on a mechanical part in a univocal manner.

ISO/TC 213 has taken the responsibility of developing a language and methods of verification associated to each specification acting in a function in the product. Within this framework, the notion of the generalized uncertainty principle has appeared. It is based on the fact that generalized uncertainty includes:

- Correlation uncertainty: it arises from the fact that the intended functionality and the controlled geometric may not be perfectly correlated.

- Specification uncertainty: it results from incorrect or incomplete application of geometric specifications.

- The measurement uncertainties in a verification process.

Concerning the expression of measurement uncertainties, the GUM [4] and associated standards offer methods to de-

* Corresponding author. Tel.: +33-4-4293-9096; fax: +33-4-4293-

9070. E-mail address: bachmann@ iut.univ.aix.fr (J. Bachmann). 
terminate their origins and of quantifying the latter (physical and chemical phenomena, mathematical modeling of the understood phenomena, interactions between materials and operating conditions).

In conformance with the new directives taken by ISO, the setting up of calculations of uncertainties in the software of Coordinate Measuring Machines (CMM) is proposed. This consists in being able to automatically display the uncertainty associated with the result.

The range of normalized specifications generally leads to the calculation of a set of distances when no modifier of the envelope condition, maximum or minimum material condition is specified. These situational characteristics are of the following type:

- Point/point distance

- Point/plane distance

- Point/line distance

In the course of this article, the concept of the Statistical Confidence Boundary (SCB) will be presented. This concept enables the user to visualize the uncertainties in normal surfaces. Secondly, the uncertainties in the case of the study of a coaxial specification will be propagated, conforming to the directives of ISO/TC 213. The use of a computerized model will allow the metrologist to provide extra information on the acceptance of the specification. This tool which aides in decision-making helps to define corrective actions on the range of measurements.

\section{3D uncertainties: optimization and representation}

Determining uncertainties is a great concern for researchers. A number of studies refer to the GUM [4], which shows the statistical bases that must be used.

Different directions, which lead to the calculation of 3D uncertainties, are distinguished. One of them concerns the study and best-fit of a set of measured points coming directly from CMM. A number of projects related to the study of a set of measured points were carried out by Henke and Summerhays $[5,6]$. They suggest methods of the evaluation of geometric errors obtained on the parts where the production process is known. The best-fit methods used take into account the characteristics of the process in order to find the best model. The least-squares criterion has been retained as best-fit method. It can calculate the extended zone model (EZN) coefficients for each of the geometric defects in the retained production method. In the same way, Kurfess and Banks [7] propose a strategy for sampling the surface of the most efficient part and a more adequate optimization with the functions of the part in order not to reject good parts or accept bad ones. Uncertainty is obtained through the knowledge of the covariance matrix. Best-fit methods are carried out numerically by algorithms which allow for different kinds of errors generated by each process type.
Choi and Kurfess [8] put forward a method of estimating the uncertainty of the measurement on envelope surfaces. They adopt a statistical approach in considering the measurement uncertainty as the stochastic noise of a point sample. This technique is similar to the one used in the evaluation of extreme fit. Results are given in a particular case where surfaces are theoretically built using a random sample method.

A number of methods, which are sometimes divergent, are used to associate a shape to a point sample. An analysis of these problems was presented by Weckenmann et al. [9]. He clearly demonstrated that the association of a surface to a set of measured points assumed the knowledge of the surface in question completely. A criterion of evaluation must be chosen in relation to the function of each surface in the part being checked. However, the least-square criterion remains the one most used because of its robustness especially when the points are sparse in the sample. Without pre-judging the distribution of the sample points (study of the roughness of a surface). Mestre and Abou-Kandil [10] demonstrated that a best-fit with the least-squares method sometimes causes a non-negligible error in the result. They put methods of Bayesian prediction to use for surface metrology.

Determining uncertainties using a vectorial modeling of surfaces based on the same model as Yau is proposed [11]. The displacement matrix obtained will allow us to use a non-linear model and will allow us to set up an analytical model for ascertaining uncertainties.

The geometric representation of uncertainties was the subject of a study done by Hernla [12] in 1993. He presents the foundations of the representation of uncertainties in 2D cases. The hypothesis retained only makes references to models where the variables are not correlated. In this case, the author wonders about the geometric shape that the confidence boundaries of a point can have when it is the result of two points having an interval of confidence. He thus approaches the question of the propagation of uncertainties. The concept of Statistical Confidence Boundaries will be presented below. It is based on the same principal but no longer requires any particular hypothesis (3D cases and correlated variables).

\section{Uncertainties : the concept of Statistical Confidence Boundary (SCB)}

\subsection{Geometric approach in surface association}

This is the approach, which is used the most. It consists in determining a theoretical surface which best passes from a set of points following a criterion: infinite norm (Tchebichev norm), norm 1 (least absolute deviation LAD) or norm 2 (least-squares norm). The best surface obtained is the one, which minimizes a criterion. If the sum of the squares of the distances of points to the plane under research is the criterion retained, then, the criterion of the least-squares method is used. The result of the association enables us to evaluate mean values of the parameters of the associated surfaces. As 


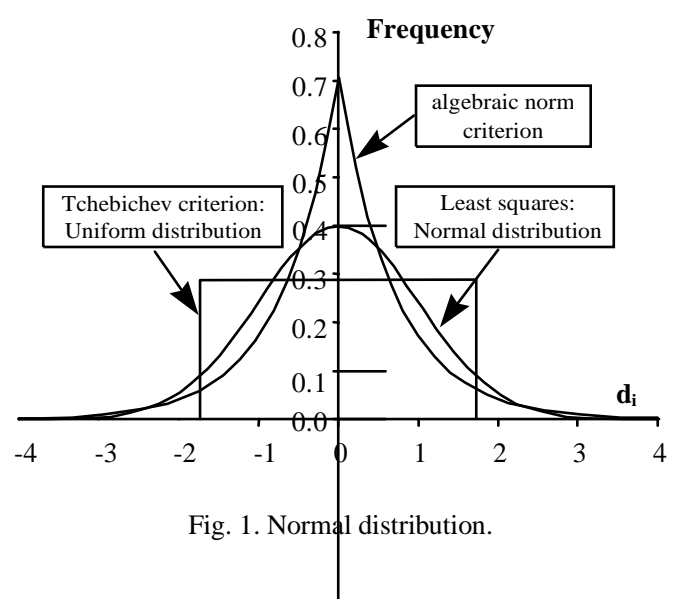

a general rule, linearizing displacement to the first order is sufficient. This amounts to us ng a translation and a rotation of the local 3D basis. The drawback to this approach resides in the fact that the uncertainty on the estimated parameters can only be obtained with an approximation. From a numeric point of view, providing initial parameters, which are close to the final solution, is indispen able [13]. Moreover, the geometric modeling of certain suffaces requires making a transfer. This is the case in the assoc ation of a straight line in space, which presently needs to be projected on two perpendicular planes (characteristics of the local 3D basis). Therefore, it is easier to use information tech hology to calculate each of the coefficients of the two straigh lines found in this manner.

\subsection{Statistical approach in surface association}

All measurements, howeve careful and scientific, are always tainted by uncertainty. Their estimation through different studies simply allows us to evaluate them, to get a rough estimate of their value, in ofder to reduce them if necessary [14]. This evaluation rests on the notion of inference: it consists in stating results (e.g. dimension) related to a phenomenon without knowing it entirely. This notion must be differentiated from the notion of deduction [15]. The latter requires setting up hypotheses, which will serve in obtaining a result. The strong hypothesi that must be verified concerns the distribution of the distances $d_{i}$, which must be compatible with the criterion, used for the association. This approach is based fundamentally on statistical mathematics (Fig. 1).

Practically speaking, the studied parameter must be considered as the population to pe characterized. Determining moments in a statistical sense can do this. It is, in fact, one of the characteristics of the population. Generally, only the mathematical expectation, or first statistical moment is determined [16].

As the surface is real, it is made up of an infinite number of points. When an operator takфs a measurement, he is in fact taking a sampling of the real surface, thus creating a sampling, made up of a set of $n$ points. From these $n$ points, CMM software associates the surfaces. Statistical theory makes the calculation of the set of all moments serving to character- ize the population. The surface characteristics (point, vector, scalar) are estimated by their mathematical expectation and by their variance. The statistical approach of surface association and the ascertainment of their uncertainty are compatible with the GUM proposals [4].

A set of digitized measured points contains a certain amount of information that must be in order to express the second statistical moment. Only in making an adequate model can automatic calculations of this information be had $[16,17]$. The uncertainty obtained is a convolution of several state variables: including machine variability, geometric error by the machine, along with the signature of the process.

\section{Vectorial description and uncertainties}

In the above paragraph, it has been shown that the notion of statistical vectors is present in 3D metrology. The following paragraph will define the properties of this statistical vector.

A statistical vector $\boldsymbol{a}$ of dimension $n$ belonging to $R^{n}$ is the vector $\left[a_{1}, a_{2}, \ldots, a_{n}\right]^{T}$. The $a_{i}$ components are statistical variables. Its cumulative distribution function $F$ of $R^{n}$ to $R$ is defined by:

$$
\begin{aligned}
& F: a_{i} \rightarrow \square \operatorname{Prob}\left(a_{i}<u_{i}\right), \quad \forall\left(a_{i}\right) \in R^{n} \\
& F\left(a_{i}\right)=\ldots \quad f\left(u_{i}\right) \mathrm{d} u_{i}
\end{aligned}
$$

With $f$ probability density associated with vector $\boldsymbol{a}$ if the statistical vector has continuous components.

The classic properties in 1D statistics are still valid:

- The density of probability $f$ has values in $R+$.

- The cumulative distribution function has a value of:

$$
F\left(a_{i}\right)=\quad \ldots \quad f\left(u_{i}\right) \mathrm{d} u_{i}=1
$$

It is assumed that the statistical vector $\boldsymbol{a}$ has continuous components with a density of probability $f$ which allows for moments.

The latter is defined by:

$m_{k_{i}}=\quad \ldots \quad u_{i}^{k_{i}} \boldsymbol{f}\left(u_{i}\right) \mathrm{d} u_{i}$

With $k_{i} \in N$ designating the first statistical moment $k$ of the variable is of vector $\boldsymbol{a}$. If the $a_{i}$ (statistical) components of the vector have an expected value, the centered moments can be calculated by:

$\mu_{k_{i}}=\quad \ldots \quad\left[u_{i}-E\left(u_{i}\right)\right]^{k_{i}} \boldsymbol{f}\left(u_{i}\right) \mathrm{d} u_{i}$

For example, in the case of an equation of a plane $u \cdot x+$ $v \cdot y+w \cdot z+h=0$, the statistical vector $a$ has the following components:

$\mathbf{a}=[u, v, w, h]^{T}$ 
The first and second statistical moments can thus be defined as:

$$
\begin{array}{ll}
m_{1,0,0,0}=E(u), & m_{0,1,0,0}=E(v), \\
m_{0,0,1,0}=E(w), & m_{0,0,0,1}=E(h) \\
\mu_{2,0,0,0}=\operatorname{Var}(u), & \mu_{0,2,0,0}=\operatorname{Var}(v), \\
\mu_{0,0,2,0}=\operatorname{Var}(w), & \mu_{0,0,0,2}=\operatorname{Var}(h)
\end{array}
$$

The moments $\mu_{1,1,0,0} ; \mu_{1,0,1,0} ; \mu_{1,0,0,1} ; \mu_{0,1,1,0} ; \mu_{0,1,0,1}$; $\mu_{0,0,1,1}$ define the terms in covariance between the variables of the statistical vector.

$$
\begin{aligned}
& \mu_{1,1,0,0}=\operatorname{Cov}(u, v), \quad \mu_{1,0,1,0}=\operatorname{Cov}(u, w), \\
& \mu_{1,0,0,1}=\operatorname{Cov}(u, h) \\
& \mu_{0,1,1,0}=\operatorname{Cov}(v, w), \quad \mu_{0,1,0,1}=\operatorname{Cov}(v, h), \\
& \mu_{0,0,1,1}=\operatorname{Cov}(w, h)
\end{aligned}
$$

The notion of covariance matrix is useful in representing the second statistical moments.

The above paragraph defines the different moments of the statistical vector under the existence hypothesis. Starting from these fundamental notions of multidimensional statistics, it is therefore possible to define the expected value and the covariance matrix of a statistical vector.

When $\boldsymbol{a}$, a $n$ dimension statistical vector and of components $\left[a_{1}, a_{2}, \ldots, a_{n}\right]^{T}$ has first statistical moments, its expected value is defined as

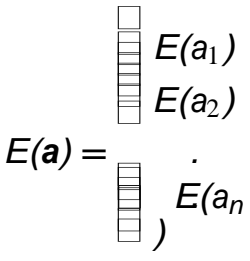

Given $\boldsymbol{a}$, a statistical vector of dimension $n$ and of components $\left[a_{1}, a_{2}, \ldots, a_{n}\right]^{T}$ which admits second statistical moments, its covariance matrix is defined as:

$$
\begin{aligned}
& \operatorname{Cov}(a)
\end{aligned}
$$

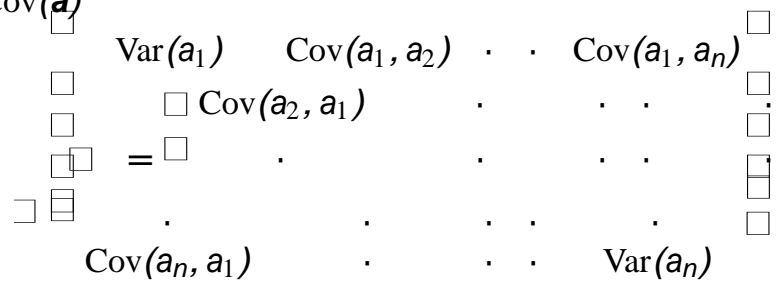

The covariance matrix is thus a square matrix of side $n$.

For example, in the case of a plane, the expected value and the covariance matrix are:

$$
\begin{aligned}
& E(a)=\text { 具长(U) } \\
& \begin{array}{l}
E(E y) \\
h)
\end{array}
\end{aligned}
$$

$$
\begin{array}{c|ccc}
\mathbf{C} & \mathrm{Cx}+/-\mathrm{U}_{\mathrm{Cx}} & \mathbf{V} & \mathrm{Vx}+/-\mathrm{U}_{\mathrm{Vx}} \\
\text { (Point) } & \mathrm{Cy}+/-\mathrm{U}_{\mathrm{Cy}} & \text { (Line) } & \mathrm{Vy}+/-\mathrm{U}_{\mathrm{V}_{\mathrm{y}}} \\
& \mathrm{Cz}+/-\mathrm{U}_{\mathrm{Cz}} & & \mathrm{Vz}+/-\mathrm{U}_{\mathrm{Vz}}
\end{array}
$$

Fig. 2. Characteristic elements.
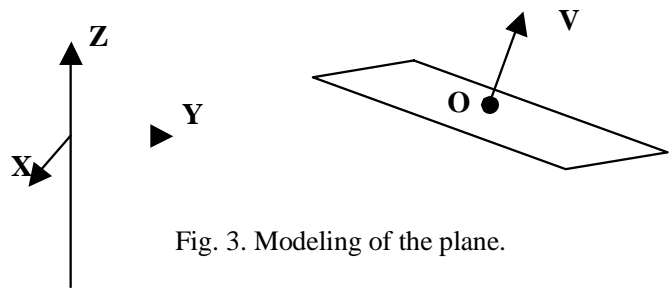

Fig. 3. Modeling of the plane.

$$
\begin{array}{ccccc}
\operatorname{Cov}(a) & & & \operatorname{Cov}(u, h) \\
\operatorname{Var}(u) & \operatorname{Cov}(u, v) & \operatorname{Cov}(u, w) & \square \\
\square & \square \text { ov }(v, u) & \operatorname{Var}(v) & \operatorname{Cov}(v, w) & \operatorname{Cov}(v, h) \\
\square \operatorname{Cov}(w, u & \operatorname{Cov}(w, v) & \operatorname{Var}(w) & \square \operatorname{Cov}(w, h \\
) \operatorname{Cov}(h, u) & \operatorname{Cov}(h, v) & \operatorname{Cov}(h, w, & \square
\end{array}
$$

The first and second statistical moments can beVarl( 2 llated, in other words, the expected value and the covariance matrix of each of the characteristic entities. The least-squares criterion is used. The modeling used is a vectorial modeling of surfaces ( $C$ is the characteristic point on the surface, $V$ the characteristic vector, $R$ and $\alpha$ two scalars or intrinsic parameters) (Fig. 2). Determining these four entities enables us to calculate the set of normal surfaces in 3D metrology in conformance with the vectorial description.

Knowing the covariance matrix facilitates the identification of the uncertainty, which was used in estimating the associated derived element. This, in turn, enables us to define the volume in which the associated derived element with given risk is found. The limit of this volume is defined with the SCB.

\section{Definition of a SCB:}

Theoretical envelope obtained from first and second statistical moments of the parameters of position and orientation of the best-fitted surface to a given risk $\alpha$.

The dimensions of the SCB of the surface in a local 3D basis are defined by eigenanalysis of the covariance matrix.

Let us take the example of a plane surface described using vectorial geometry by a vector and a point. The vector and point entities are characterized by three components, which are, in a 3D basis ( $x$-abscissa, $y$-ordinate, $z$-coordinate) (Fig. 3 ). Each component is estimated using a numerical value (mean value). The covariance matrix links these statistical components. It is used to characterize the uncertainty associated with each component.

\section{Visualizing the results}

With this concept the surface is no longer considered as uniquely determined but rather as belonging to a spacing zone limited by a defined envelope with a given risk. In the fol- 
Table 1

Results (confidence ratio $k=2$ )

$\begin{array}{llll}\mathrm{Ox} & -4.240 \mathrm{E}+01 & +/- & 1.818 \mathrm{E}-06 \\ \mathrm{Oy} & 6.285 \mathrm{E}-02 & +/- & 7563 \mathrm{E}-06 \\ \mathrm{Oz} & 1.511 \mathrm{E}-02 & +/- & 5062 \mathrm{E}-03 \\ \mathrm{Nx} & 3.592 \mathrm{E}-04 & +/- & 8976 \mathrm{E}-05 \\ \mathrm{Ny} & 1.494 \mathrm{E}-03 & +/- & 1077 \mathrm{E}-04 \\ \mathrm{Nz} & 1.000 \mathrm{E}+00 & +/- & 1639 \mathrm{E}-07\end{array}$

lowing sections, the example of a simple numerical will be considered. A plane has been drawn with 16 randomly set points using a measuring machine equipped with a Renishaw type TP2 touch trigger probe, in the measuring conditions defined by the manufacturer. The characteristics of the asso-

\section{ELS Point}

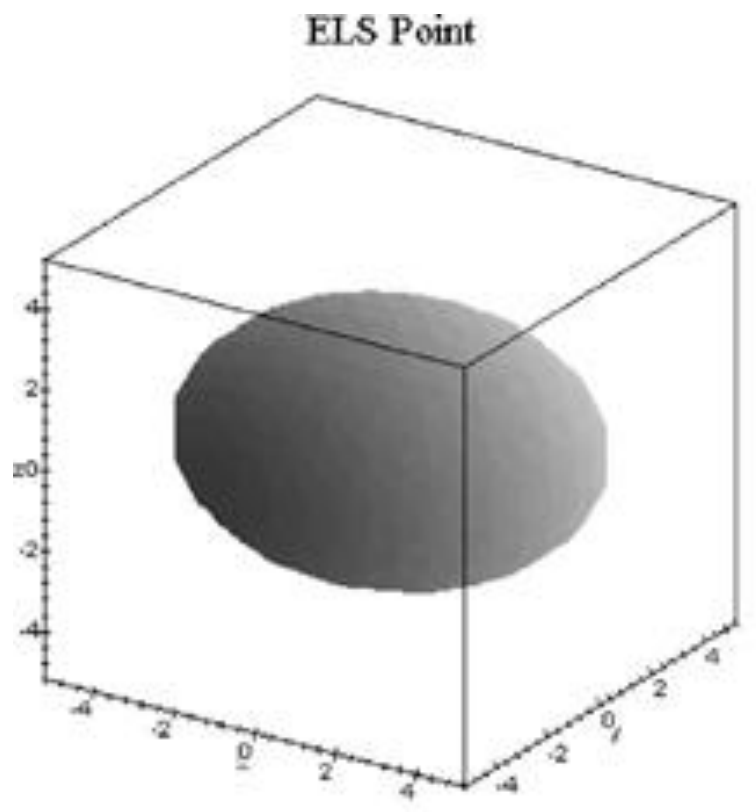

ciated surface are shown in Table 1 (the six components are linked statistically).

Table 1 displays the results of the associated surface with a confidence ratio of $k=2$ which is necessary in framing mean values. The table is made up of two columns:

- The first one proposes an estimate of the mean ( $\mathrm{mm})$. The results are typical and conform to those found with the MMT software.

- The second one presents the uncertainty with a confidence ratio of $k=2$. This corresponds to a new piece of information, which is not currently offered.

The limits of the zones of this surface are calculated from the covariance matrix. This space can be visualized from data

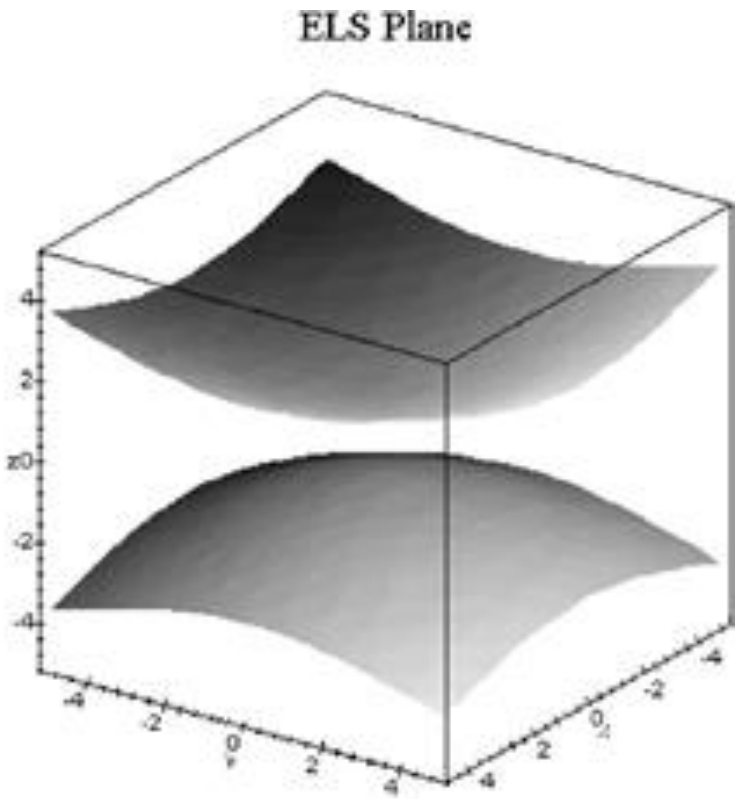

\section{ELS line}

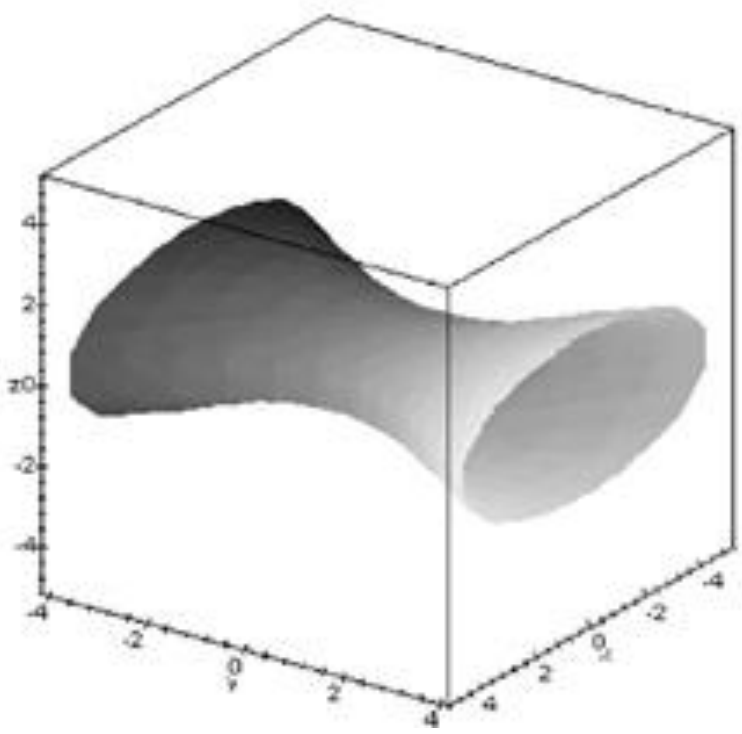

Fig. 4. Presentation of the SCB point, plane and line. 
points. For the point, the envelope is elliptical. For the plane, there are two symmetric envelopes in relation to the mean surface. It is noted that uncertainty rises with the distance of the point considered at the centroid (Fig. 4).

To make a representation in SCB form, the covariance matrix (order 2 tensor) is used. This tensor can be characterized by a conical whose features do not depend on an axis system. For instance, the point position resolution can be characterized by an elliptical of the following equation:

$$
\frac{X^{2}}{1 / \sigma_{X}}+\frac{Y^{2}}{1 / \sigma_{Y}}+\frac{Z^{2}}{1 / \sigma_{Z}}=1
$$

The standards deviation $\sigma_{X}, \sigma_{Y}$, and $\sigma_{Z}$ are obtained from the covariance matrix expressed in a local $3 \mathrm{D}$ axis (matrix eigenanalysis).

The SCB characterizes the doubt with which the mean is estimated. For the plane and the line, the associated surface (or mean) is the functional surface. This is not the case for the other surfaces in general:

- For the cylinder or the cone, the mean value characterizes the axis.

- For the sphere and the circle, the mean value characterizes the center. Indeed, for the sphere, SCB limits the point positions, which are characteristic of the associated surface and in no way the associated surface.

\section{The propagation of uncertainties}

From the SCB to the Statistical Generated Object (SGO). During the verification phase, a process measurement will be established. When calculating each associated derived feature, an SCB can be determined. In order to be able to estimate the influence of these SCB on the resulting SCB, mathematical formalism must be put in place. It is suggested to use the SGO to this effect. The SCB are the geometric representation of the zone of uncertainty calculated from a covariance matrix. In order to have a synthetic object, the SGO will be defined.

For random vector of three components, the SGO is a matrix of dimension $(6,4)$, which gathers the first and second statistical moments.

The SGO of each elementary surface or the result of an operation between two SGOs will be defined from basic SGOs corresponding to intrinsic parameters (vector and scalar element). Table 2 above represents the basic SGO of a point or a vector.

Table 2

Statistical generated object

$\begin{array}{llll}\text { Ex } & 0 & 0 & 0 \\ \text { Ey } & 0 & 0 & 0 \\ \text { Ez } & 0 & 0 & 0 \\ 0 & \operatorname{Var}(X) & \operatorname{Cov}(X, Y) & \operatorname{Cov}(X, Z) \\ 0 & \operatorname{Cov}(X, Y) & \operatorname{Var}(Y) & \operatorname{Cov}(Z, Y) \\ 0 & \operatorname{Cov}(X, Z) & \operatorname{Cov}(Z, Y) & \operatorname{Var}(Z)\end{array}$

In the example of the associated-derived cylinder, the SGO is obtained during the association phase. It will be the assembling of two basic SGOs of the vector type and of a basic SGO of the scalar type.

\section{Propagation}

The propagation procedure uses the SGOs obtained during the association phase. This stage is essential for initiating propagation.

\subsection{Definition of propagation}

It is the possibility of creating a SGO from other SGOs. The SGO obtained in this way must be minimal (the determinant of the covariance matrix of the propagated parameters must be minimal).

The question is to determine the SGO resulting from the beginning SGO for each of the calculated surfaces, i.e. to be able to trace the SCB.

From a statistical point of view, the question is to determine the second statistical moment at the studied parameter in relation to the entry parameters. Fig. 5 illustrates propagation by placing a sensibility factor. The studied parameter is a scalar and only depends on a unique entry $x$ variable. The second statistical moment of the exit parameter is a function of the local gradient calculated by the partial derivative of the function at the studied point [17]. This function is generally known. It can, however, be implicit but it is at any rate solvable thanks to numerical methods [18].

For an exit variable depending on several entry variables, the propagation principle is identical [19].

$$
\begin{aligned}
& \operatorname{Var}(y)={ }_{\overline{\partial x}}^{\frac{\partial y^{2}}{\partial x}} \operatorname{Var}\left(x_{i}\right) i=1
\end{aligned}
$$

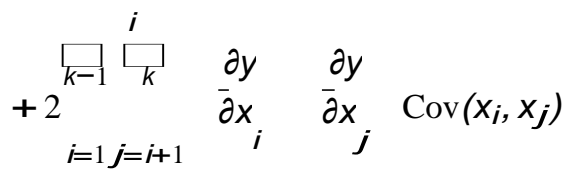

For a scalar parameter $y$ depending on several variables of dimension $n$, variance can be obtained with:

$\operatorname{Var}(y)=J_{y} \operatorname{Cov}(A) J_{y}^{T}$

where $\operatorname{Cov}(A)$ represents the covariance matrix of $n$ entries parameters $x_{i}$ and $J_{y}$, the Jacobian matrix defined by:

$\forall i \in\{1 \ldots n\}, \forall p \in\{1 \ldots n\} \forall x_{i}$

$\in P_{p}$

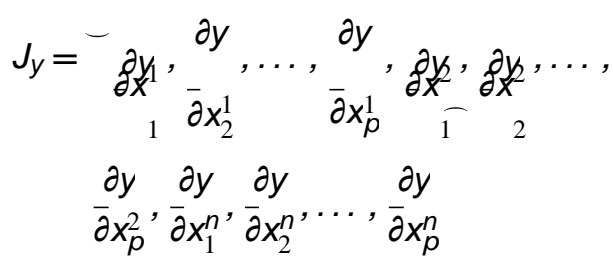




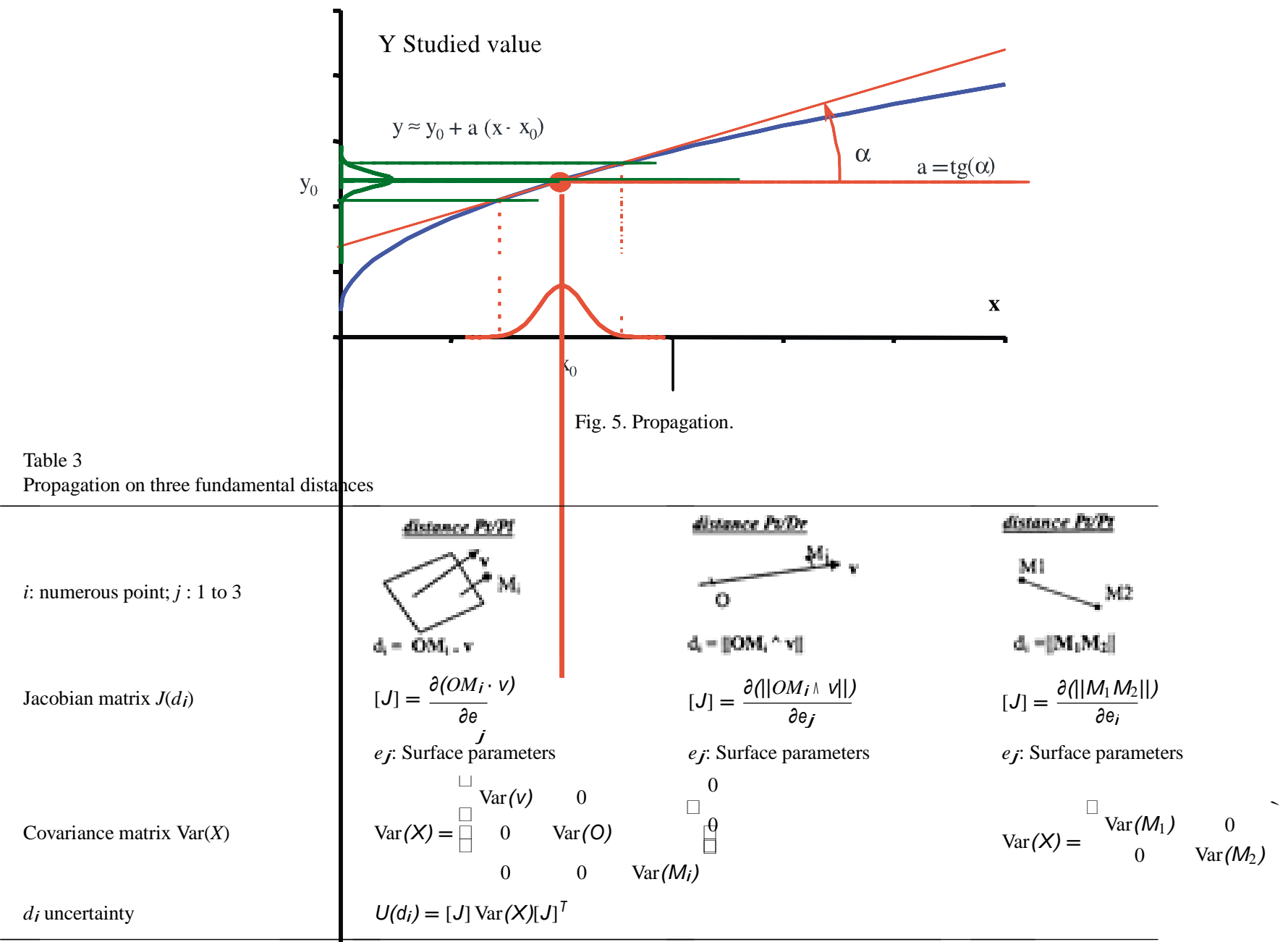

Table 3 gives an example of propagation applied to the calculation of three fundamental d stances.

In 3D measurement, the studied parameter is a vector. Thus, the second statistical monent is homogeneous in a covariance matrix of dimension 3. This is the SGO of the studied parameter.

Determining the final SGO requires setting up a process. Calculating a SGO from two S\&Os is done via a set of propagation modulus, which are function of the constructions needed to check a specification

Mathematical operations betyeen SGOs can be carried out with these modules. This corr sponds to geometric operations, which allow for the creatidn of a SCB from other SCBs. For example, the Projected Point SCB module on Plane SCB enables us to create a SCB Point.

\subsubsection{Example}

Let us consider the case of a geometric tolerance of position: a coaxiality (Fig. 6).

7.1.1.1. Interpretation. The specified cylinder axis must be included in a cylindrical zone of diameter $t$, coaxial to the axis of the referenced surface.
The geometric elements considered are two non-ideal surfaces (S1) and (S2) which are nominally cylindrical and identified by a partition and filtration operation taken from a "skin model."

a. A best-fitted surface of a cylindrical type (CY1) comes from a set of points (S1) with an objective of minimizing the sum of the squares of the distances from the $\mathrm{S} 1$ points to the cylinder (CY1). The axis of cylinder S1 is defined by a series of the following mathematical operations (Fig. 7).

b. A set of ideal constructed $i$ planes perpendicular to the CY1 axis is considered (Fig. 8).

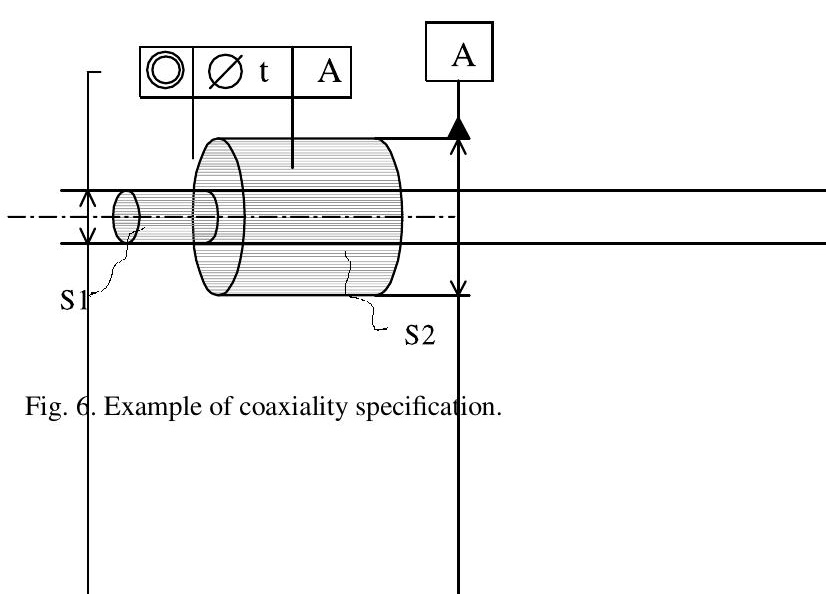




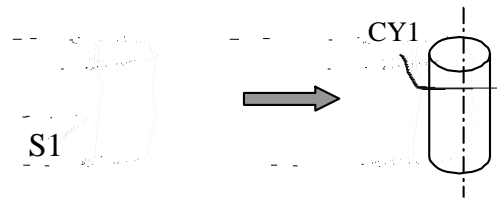

Fig. 7. First step.

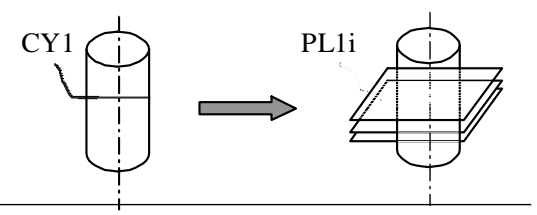

Fig. 8. Second step.

c. In each of these planes (PL1i), the nominally circular lines are considered. A best-fitted operation must be carried out in order to obtain $i$ circles (CE1i) in each of the planes (PL1i) (Fig. 9).

d. Next a surface is best fitted whose reference is a cylindrical type (CY2) from the surface (S2).

In order to meet specifications, the greatest distance value $d_{i}$ (CE1i, CY2) must be inferior to half the tolerance $t$ $\left(\max \left(d_{i}\right)<t / 2\right)$.

In this example, it is clearly illustrated that the calculations of distances come after a long series of operations, which is necessary in order to respect the directives (orders, instructions) of the ISO/TC 213 group.

Propagation of the uncertainties of any geometric specifications that follow the principle of independence (without modifiers) ean be done via the software program. It is not linked to a set of particular operations.

The following paragraph will deal with this coaxiality in a numerical point of view. The CMM programming is initiated according to the range given above. The CMM is only used as a means of acquiring points on real surfaces. Any 3D measurement machine could have also been used such as laser Tracker or coaxial optical captor. The data used can be of any kind as long as it contains a series of three coordinates $(X, Y, Z)$.

The result of the specification to be studied inherits the value of the uncertainties of the beginning digitized surfaces. Thus, first of all, the results of the uncertainties for each of the surfaces will be shown.

The specification requires a definition of a datum surface. In our example, this is the axis of the cylindrical surface (Fig. 10).

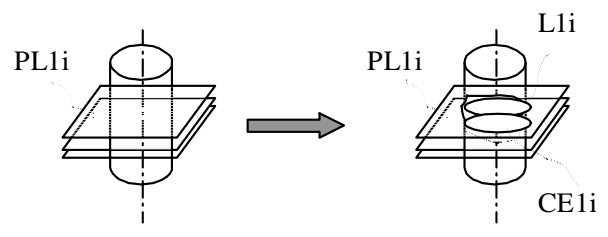

Fig. 9. Third step.

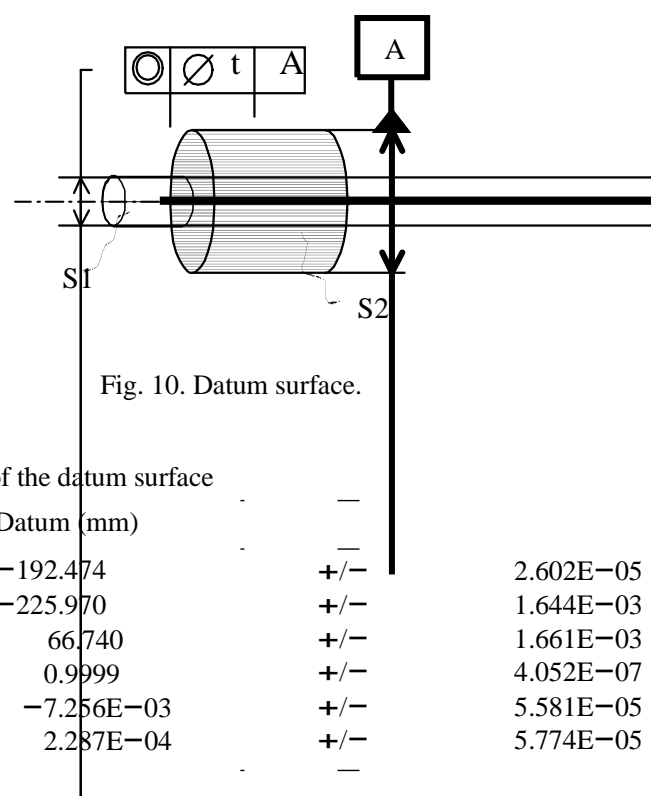

The axis of the surface, after fitting, is defined by a characteristic point (three coordinates and their respective uncertainties) as well as the directing vector of the axis (three coordinates + their respective uncertainties): Table 4 .

As for the specified surface, four circles have been digitized. Thus, an image of the real axis of the specified cylinder has been calculated, as well as the geometric characteristics of the fitted surfaces for each of these four circles.

Table 5

Characteristics of the cifcles representing the specified surface Circle $1(\mathrm{~mm})$

$\begin{array}{lc}\mathrm{Ox} & -249.4645 \\ \mathrm{Oy} & -225.5520 \\ \mathrm{Oz} & 66.7315 \\ \mathrm{Nx} & 0.9999 \\ \mathrm{Ny} & -7.475 \mathrm{E}-03 \\ \mathrm{Nz} & 4.244 \mathrm{E}-04\end{array}$

$+/-$

3.476E-05

$+/-\quad 4.641 \mathrm{E}-03$

$+/-\quad 4.640 \mathrm{E}-03$

$+/-\quad 1.491 \mathrm{E}-06$

$+/-\quad 1.992 \mathrm{E}-04$

$\begin{array}{ll}+/- & 1.992 \mathrm{E}-04 \\ +/- & 1.991 \mathrm{E}-04\end{array}$

Circle $2(\mathrm{~mm})$

$\begin{array}{lc}\mathrm{Ox} & -229.465 \\ \mathrm{Oy} & -225.702 \\ \mathrm{Oz} & 66.738 \\ \mathrm{Nx} & 0.999 \\ \mathrm{Ny} & -7.440 \mathrm{E}-03 \\ \mathrm{Nz} & 4.229 \mathrm{E}-04\end{array}$

$+1-$

$3.761 \mathrm{E}-05$

$5.047 \mathrm{E}-03$

$5.046 \mathrm{E}-03$

$1.700 \mathrm{E}-06$

$2.281 \mathrm{E}-04$

$2.280 \mathrm{E}-04$

Circle $3(\mathrm{~mm})$

$\begin{array}{lc}\mathrm{Ox} & -209.466 \\ \mathrm{Oy} & -225.847 \\ \mathrm{Oz} & 66.742 \\ \mathrm{Nx} & 0.999 \\ \mathrm{Ny} & -7.440 \mathrm{E}-03 \\ \mathrm{Nz} & 4.170 \mathrm{E}-0\end{array}$

$3.016 \mathrm{E}-05$

$4.048 \mathrm{E}-03$

$4.047 \mathrm{E}-03$

$1.678 \mathrm{E}-06$

$2.2517 \mathrm{E}-04$

$2.251 \mathrm{E}-04$

Circle $4(\mathrm{~mm})$

$\begin{array}{lc}\text { Ox } & -169.467 \\ \mathrm{Oy} & -226.139 \\ \mathrm{Oz} & 66.751 \\ \mathrm{Nx} & 0.999 \\ \mathrm{Ny} & -7.416 \mathrm{E}-03 \\ \mathrm{Nz} & 4.086 \mathrm{E}-04\end{array}$

$2.833 \mathrm{E}-05$

$3.814 \mathrm{E}-03$

$3.813 \mathrm{E}-03$

$1.877 \mathrm{E}-06$

$2.528 \mathrm{E}-04$
$2.527 \mathrm{E}-04$ 
Table 6

Results of the four distances with their uncertainties

$\begin{array}{lll}\text { Distance } & \text { Uncertainty } & U(d)=k \times \text { uncertainty } \\ 0.00437 & 0.04699 & 0.09397 \\ 0.00101 & 0.03068 & 0.06137 \\ 0.00051 & 0.01421 & 0.02841 \\ 0.00081 & 0.00318 & 0.00636\end{array}$

Table 5 groups the results of the four circles and their uncertainties.

\section{Results in distance}

In accordance with the above paragraph, interpreting specifications requires calculating distances. Table 6 gives the result of the four point/line distances.

If only the mean value distances are considered, the part will be declared in conformity for any interval of a tolerance greater than $4.37 \mathrm{~mm}$. Columns 2 and 3 present the uncertainty and enlarged uncertainty with a confidence ratio of $k=2$. This corresponds to a unilateral risk of $97.7 \%$.

\section{Aide in decision-making}

Mechanical parts have always obtained acceptance through the knowledge of mean values. The capability of the procedure is only generally known for CMMs, by methods based on the experience of metrologists and on a repetition of measurements. This uncertainty is applied in an empirical way and is systematically a function of the precise formula of a CMM of the form: $+/-(a+b L)$. Knowledge of propagated uncertainties on a result enables us to better define the limits of acceptance.

The following paragraph gives evidence of the different types of results that can be obtained on the acceptance of a part. When having an estimation of measurement quality is not possible, the major difficulty is in accepting defective parts and refusing correct parts.

Let's consider a tolerance interval defined by a greater tolerance Ts and a lesser tolerance Ti. The measure is accepted when it is situated in this tolerance interval (Fig. 11).

$$
1
$$
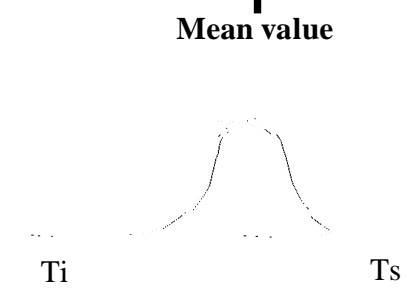

Fig. 11. Characteristics of a tolerance interval.

If the uncertainty is estimated, it can be represented graphically using a Gaussian law [20]. Indeed, the convolution of a large number of entry parameters characterized by a given distribution induces a Gaussian distribution of the studied parameter (Fig. 12).

Several cases (Fig. 13) can thus be presented as a function of the value of typical difference which characterizes the law. When the measure is contained in the tolerance interval, the part is generally declared in conformance. The metrologist cannot simply make a decision as to the acceptance of the part if uncertainty is not estimated.

When the measure is not contained in the tolerance interval, the part is usually declared in non-conformance.

The model offers an aide to the operator. From the files of digitized surfaces, the set of distances, which influence decisions on conformance, can be calculated. The uncertainty associated with each distance can be represented graphically which gives information about the chosen risk. Fig. 14 presents the results from the aide in decision-making software program. The two right and left limits define the area of the tolerance zone. Four distances are calculated. For each of these, the position of the mean value $\left(d_{1}, d_{2}, d_{3}, d_{4}\right)$ is depicted. In view of the chosen interval tolerance, the coaxiality specification is respected with regard to the mean values. The user truncates the functions of distribution at a chosen risk. (For our example, a unilateral risk of $2.5 \%$ was chosen.) The unilateral risk is obtained with a form defect law when the mean value of the distance is less than three times the uncertainty of the distance. Its cumulative density remains, however, the same than the Gaussian law's one if the unilateral condition is assumed. The latter can thus give the operator quantitative information as to exceeding the interval tolerance (Fig. 14).

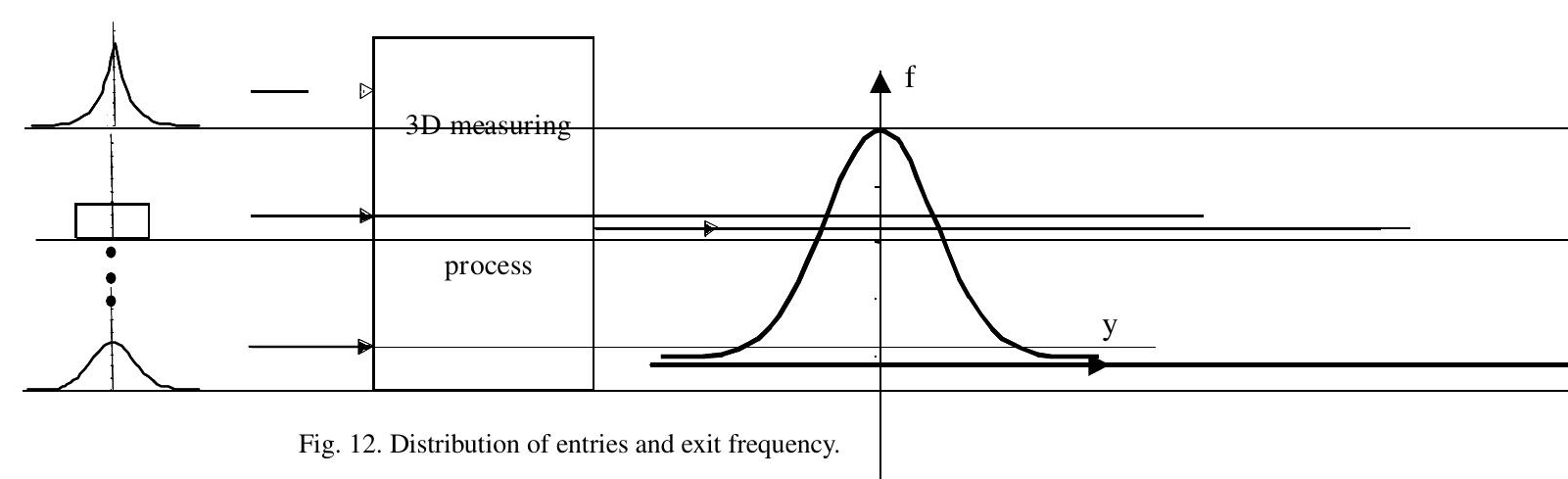




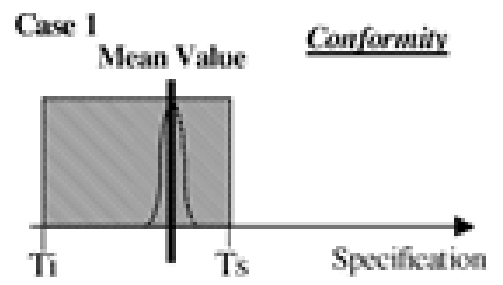
- The measuremere belongs in the tolerance interval

- The part can bed eclared in conformance with no risk

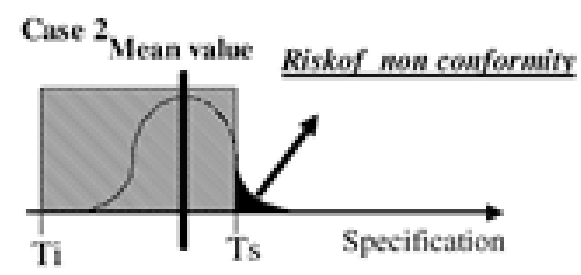

- The measurement belongs in the tolerance inacrval

- There is a risk of non conformity

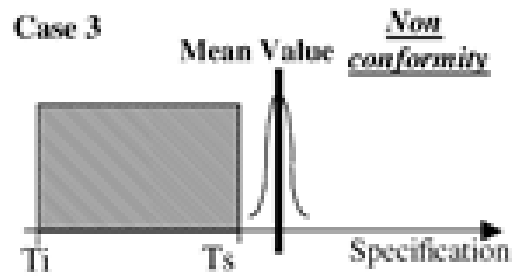

- The measurement is not contained in the tolerance interval

- The part is non conformance

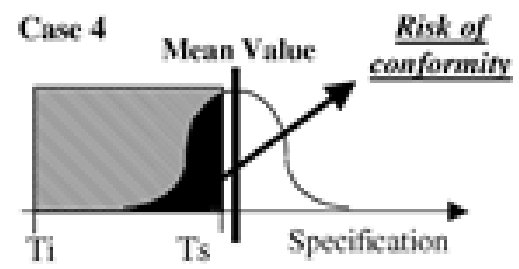

- The measurement belongs in the tolerance interval

- There is a risk of conformity

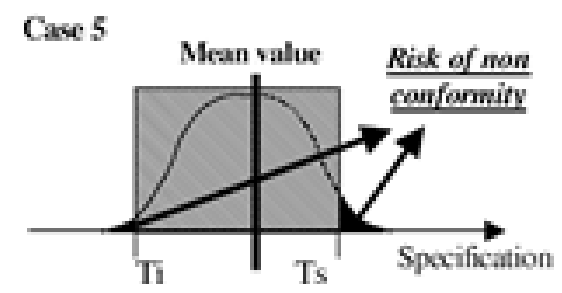

- The mearsurement is within the tolerance interval

- There is a risk of non conformance

Fig. 13. Different cases of acceptance risk or refusal.

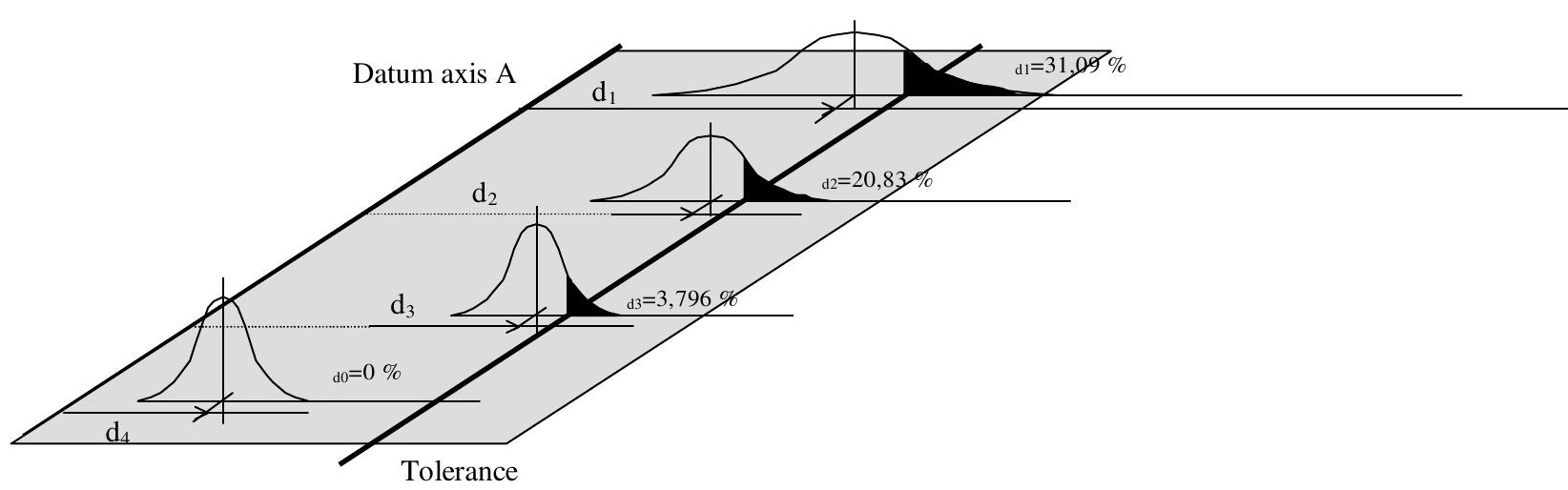

Fig. 14. Aid in decision-making (representation of the results). 
The amount exceeding the upper limits of the tolerance interval is calculated as a percentage. Thus, $d_{4}$ does not pose a problem for acceptance. However, for value $d_{1}$ there is a $31.09 \%$ risk that the mean value goes beyond the upper limit of the tolerance interval.

The operator can thus make his decision in consequence, taking corrective action aiming at reducing the obtained uncertainty. He will be able to act directly on the part checking program, modifying it, or increasing the number of points of the concerned surface in order to reduce uncertainty. The software accompanies the operator in his decision. This depends in fact on a number of parameters (price of the part, cost of measuring on the CMM, software possibilities).

\section{Conclusion}

The article has shown how the knowledge of uncertainty can be used. This method is based on statistical concepts, which are in accordance with "The Guide to the expression of the uncertainty in measurement" (GUM). It also enables us to generate uncertainties on the verification of ISO specifications (or specs in the ISO directives). The final result of the specification is a direct consequence of the actions taken by the CMM operator. Mastering the risk of acceptance or refusal enables us to inform the operator in a quantitative way. He can thus take one or more corrective actions relating to the characteristics of the product and of the enterprise. This software aide lies in the scope of a process of improving quality and conforms to the directives of the authorities of the standards organization.

\section{References}

[1] Srinivasan V. An integrated view of Geometrical Product Specification and Verification. In: Proceedings of the seventh CIRP seminar on computer-aided tolerancing, Cachan, France; 2001. p. 7-17.

[2] Dovmark J. New interesting concepts from ISO/TC 213. In: ACMC annual general meeting in Ottawa, Canada, December 3, 2001.

[3] Ballu A, Mathieu L, Dantan JY. Global view of geometrical specifications. In: Proceedings of the seventh CIRP international seminar on computer-aided tolerancing Cachan, France. p. 19-30.
[4] BIPM, IEC, ISO, IUPAC, IUPAP, OIML. Guide to the expression of the uncertainty in measurement. 1st ed. ISBN 92-6710188-9; 1993.

[5] Summerhays KD, Henke RP, Balwin JM, Cassou JM, Brown CW. Optimizing discrete point sample patterns and measurement data analysis on internal cylindrical surfaces with systematic form deviations. Precision Eng 2002;26:105-21.

[6] Henke RP, Summerhays KD, Balwin JM, Cassou JM, Brown CW. Methods for evaluation of systematic geometric deviations in machined parts and their relationships to process variables. Precision Eng 1999;23:273-92.

[7] Kurfess T, Banks DL. Statistical verification of conformance to geometric tolerance. Comput Aided Des 1995;27/5:353-61.

[8] Choi W, Kurfess T. Uncertainty of extreme fit evaluation for three-dimensional measurement data analysis. Comput Aided Des 1998;30/7:549-57.

[9] Weckenmann A, Eitzert H, Garmer M, Weber H. Functionality-oriented evaluation and sampling strategy in coordinate metrology. Precision Eng 1995;17:244-52.

[10] Mestre M, Abou-Kandil H. Measuring the errors of form of industrial surfaces: prediction and optimisation. Precision Eng 1994;16:268-75.

[11] Yau HT. Evaluation and uncertainty analysis of vectorial tolerances. Precision Eng 1997;20:123-37.

[12] Hernla M. Calculation of measuring uncertainty with CMMs under industrial conditions. In: Proceedings of third CIRP international seminar on computer-aided tolerancing, Cachan, France; 1993. p. 171-7.

[13] Kruth JP, Weiyin MA. Parameter identification of geometric elements from digitized data of coordinate measuring machines. In: Proceedings of the 29th international Matador conference, Manchester, Grain Britain; 1992. p. 277-85.

[14] Arri E, Cabiati F, D’Emilio S, Gonella L. On the application, Guide tc the expression of the uncertainty in measurements to measuring instruments. Measurement 1995;16/1:51-7.

[15] Tyler Estler W. Measurement as inference: fundamental ideas. Ann CIRP 1999;48/2:611-32.

[16] Sprauel JM, Linares JM, Bourdet P. Contribution of nonlinear optimization to the determination of measurement uncertainties. In: Proceedings of the seventh CIRP seminar on computer-aided tolerancing, Cachan, France; 2001. p. 285-91.

[17] Goch G, Tschudi U. Universal algorithm for the alignment of sculptured surfaces. Ann CIRP 1992;41/1:507-10.

[18] Cox MG. Measurement uncertainty and the propagation of distributions. In: Proceedings of the 10th international metrology congress, CD-ROM, St. Louis, France; 2001.

[19] Tuninski V, D’Emilio S, Cabiati F. Concept and rules in the guide to the expression of uncertainty. In: Metrology 99. Proceedings of the ninth international metrology congress, Bordeaux, France; 1999. p. 361-4.

[20] de Vicente J. A generalization of the law of propagation of uncertainty. An example: determining screw thread uncertainty. In: Proceedings of the eighth international metrology congress, Besançon, France; 1997. p. $369-74$. 